\title{
Drift chambers in BM@N Experiment
}

\author{
Ján Fedorišin ${ }^{1, \star}$ \\ ${ }^{1}$ Veksler and Baldin Laboratory of High Energy Physics, Joint Institute for Nuclear Research, 141980 Dubna, \\ Moscow region, Russia
}

\begin{abstract}
Drift chambers (DCH) constitute an important part of the tracking system of BM@N experiment designed to study the production of baryonic matter at the Nuclotron energies.

The method of particle hit and track reconstruction in the drift chambers is proposed and tested on the BM@N deuteron beam data.

In first step, the radius vs drift time calibration curve is estimated and applied to calculate DCH hit closest approach coordinates. These coordinates are used to construct hits in each DCH under the assumption of track linearity. Hits in both the DCHs are subsequently aligned and fitted to produce global linear track candidates. Eventually the hit and track reconstruction is optimized by the autocalibration method. The coordinate resolutions are estimated from Gaussian fits of the DCH hit residual spectra for different DCH planes. Furthermore, the deuteron beam momentum value is reconstructed in order to check reliability of the employed track reconstruction algorithm.
\end{abstract}

\section{Introduction}

BM@N (Baryonic Matter at Nuclotron) [1] is a fixed target experiment at the JINR NICA-Nuclotron complex proposed to study the hot compressed nuclear matter produced in collisions of relativistic heavy nuclei with emphasis on strange matter production. The detailed description comprising both the physical goals and the experimental apparatus of BM@N experiment can be found in $[1,2]$ or $[3]$. The outer tracker including two identical drift chambers DCH1 and DCH2 [3, 4] is placed outside the magnetic field of SP41 dipole magnet [5]. Each DCH consists of four segments measuring different track coordinates in a transverse plane relative to the beam axis. The measured coordinates are $y, x, u, v$ where $u$ and $v$ are $y$ coordinates rotated by angles $\pm 45^{\circ}$ around $z$ axis. Actually, the directly measured experimental observables are the electron drift times that are subsequently by the proper calibration technique [3, 6-8] converted to the distances of closest approach (DCA) of tracks to the anode wires. The typical drift time spectrum for the BM@N deuteron beam data along with the corresponding radius-time (or $r-t$ ) calibration curve is shown in figure 1 . Once the $y, x, u, v$ coordinates are calculated using the DCAs provided by the calibration, the missing transverse coordinates in all the DCH planes are evaluated by solving the set of linear equations as described in [3]. Finally, the track candidates from both the drift chambers are combined and fitted by straight lines to produce the global track candidates.

\footnotetext{
${ }^{\star}$ e-mail: fedorisin@jinr.ru
} 

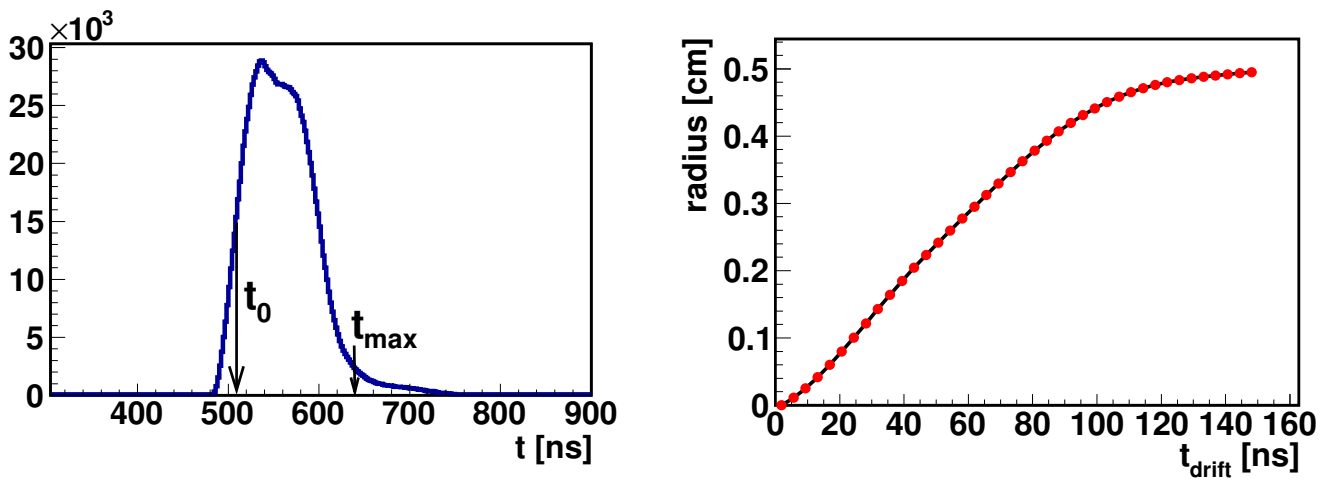

Figure 1. Experimental drift time spectrum for deuteron beam data (left) and the corresponding $r-t$ calibration curve (right).

\section{DCH autocalibration}

At the end of the track reconstruction procedure, we construct the dependencies of hit residuals $\Delta$ on drift times for all the DCH planes where the residuals are defined as

$$
\Delta=\left|r_{\text {fitted }}-r_{\text {anode }}\right|-\left|r_{\text {measured }}-r_{\text {anode }}\right|,
$$

providing that the substitute symbol $r$ represents $y, x, u, v$ coordinates in all the four DCH planes respectively. The initial $\Delta$ vs drift time spectra are averaged over the residual axes and the resulting distributions are subtracted from the corresponding calibration curves in order to suppress their systematic uncertainties. The corrected calibration curves are applied again to the experimental data and the whole tuning process including

1. calibration;

2. DCH alignment;

3. hit estimation;

4. track candidates fitting;

5. residual estimation

is iteratively repeated until the averaged residual spectra are minimized, i.e. the mean values of averaged $\Delta$ distributions converge to 0 .

Figure 2 presents the dependencies of hit residuals $\Delta$ on drift times for all the DCH1 planes after the last iteration. The analogous plots are constructed for $\mathrm{DCH} 2$ as well but they are not presented in this paper.

The averaged $\Delta$ distributions after the first and the last iterations for both the drift chambers are presented in figure 3 where the shown statistics parameters correspond to the last iterations. The spectra prove that the hit residuals are gradually suppressed during the iteration process.

\section{Results}

The hit residual spectra obtained by integration of figure 2 distributions along drift time axes are presented in figure 4. Standard deviations $\sigma$ of Gaussian fits of the residual distributions suggest the 

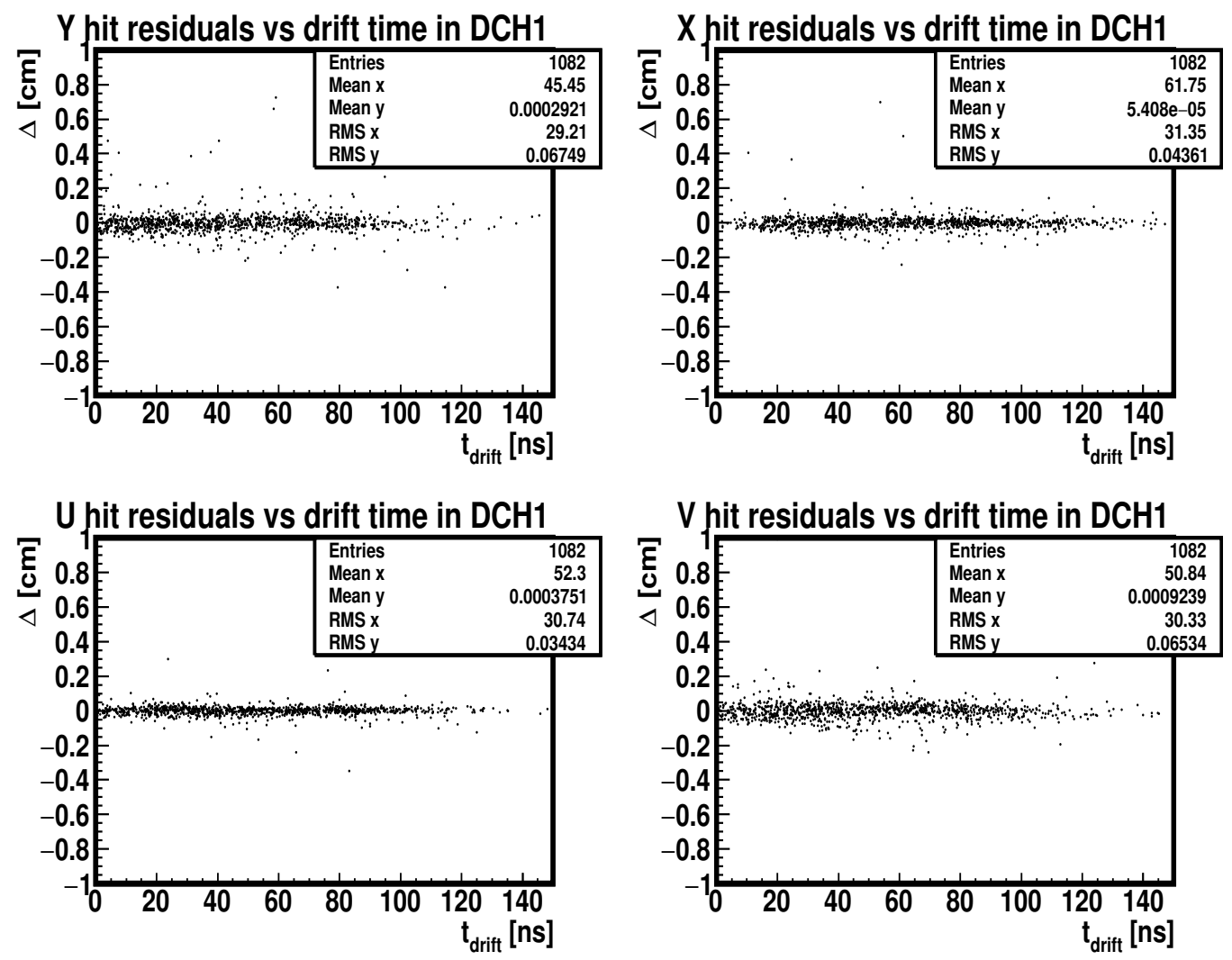

Figure 2. Hit residuals dependence on drift time for all the DCH1 coordinate planes after the last iteration.

coordinate resolutions in all the DCH1 planes. The coordinate resolutions for all the planes of DCH1 and $\mathrm{DCH} 2$ are listed in table 1. Taking into account that the arrangement of $\mathrm{DCH}$ planes along $z$ axis

Table 1. Coordinate resolutions of all the DCH planes.

\begin{tabular}{|c|c|c|c|c|}
\hline & $\mathrm{V}[\mu \mathrm{m}]$ & $\mathrm{U}[\mu \mathrm{m}]$ & $\mathrm{X}[\mu \mathrm{m}]$ & $\mathrm{Y}[\mu \mathrm{m}]$ \\
\hline DCH1 & 350 & 160 & 330 & 330 \\
\hline DCH2 & 570 & 330 & 270 & 540 \\
\hline
\end{tabular}

follows the order $\mathrm{V}, \mathrm{U}, \mathrm{X}, \mathrm{Y}$, one can see that the coordinate resolutions are better for inner DCH planes $(\mathrm{U}, \mathrm{X})$ than for the outer ones $(\mathrm{V}, \mathrm{Y})$. Furthermore, the tracks in DCH1 are reconstructed with higher precision than in $\mathrm{DCH} 2$, which can be explained by higher efficiencies of $\mathrm{DCH} 1$ planes as well as by the additional error brought to $\mathrm{DCH} 2$ track coordinates through the detector alignment procedure.

Evolution of the iterative process is demonstrated in figure 5 showing the dependence of Gaussian $\sigma$ of residual spectra on iteration number for both the drift chambers: It is obvious that the saturation is achieved very fast, just after 2-3 iterations. However, the coordinate resolution does not get significantly improved by the autocalibration procedure since the observed coordinate error decreases only about $15 \%$. 

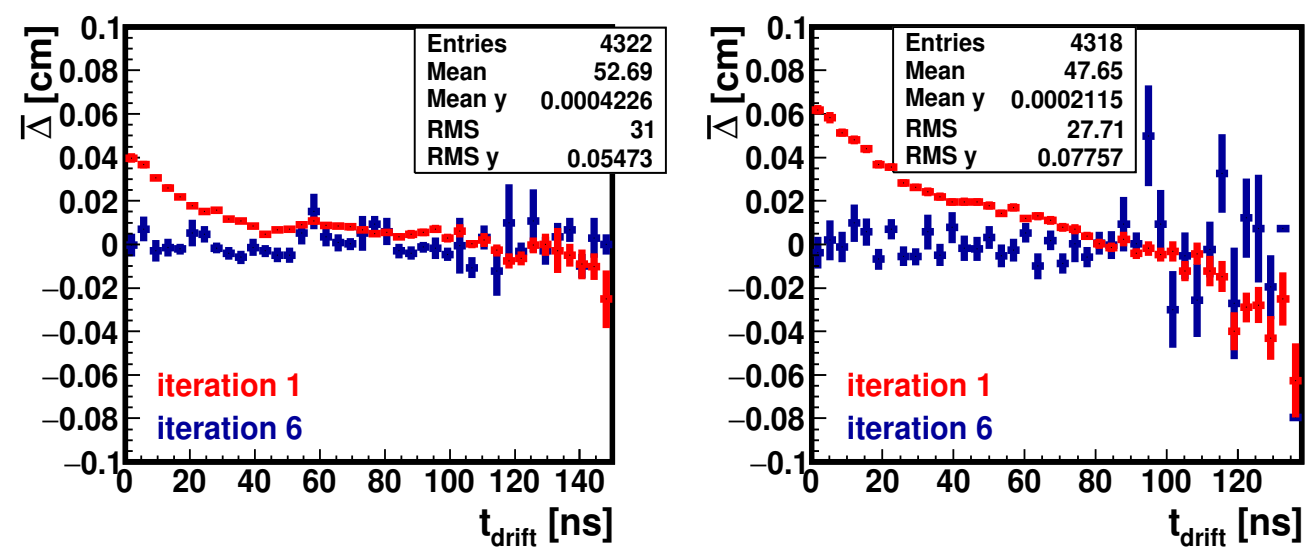

Figure 3. Averaged hit residuals $\bar{\Delta}$ for DCH1 (left) and DCH2 (right).

\section{Consistency check of the track reconstruction algorithm}

The DCH track reconstruction algorithm is verified through the momentum reconstruction of deuteron beam. In general if beam moves along $z$ axis, its deflection in homogeneous magnetic field in $x z$ plane is described by angle $\varphi_{x z}$ which depends on magnetic field integral and beam momentum $p$ :

$$
\varphi_{x z}(\mathrm{rad})=q \frac{\int B d l}{p}
$$

$$
\Longrightarrow \quad p=q \frac{\int B d l}{\varphi_{x z}},
$$

where $B$ is $B_{y}$ component of magnetic field and $q$ is beam particle charge [9]. Figure 6 displays the dependencies of angle $\varphi_{x z}$ and beam momentum $p$ on magnetic field integral. The different values of $\varphi_{x z}$ and $\int B d l$ come from the different investigated runs. The presented dependencies on the left and on the right agree with the functions 2 and 3 respectively. The figure 6 on the left shows also the extrapolated deflection angle $\varphi_{x z}$ for $B=0$ which is interpreted as an inclination angle of DCH transverse planes and therefore subtracted from all the $\varphi_{x z}$ angles in 3 employed to estimate the beam momentum $p$. The final value of deuteron momentum is thus $8.4 \pm 0.1 \mathrm{GeV} / \mathrm{c}$ which is somewhat lower than nominal value $8.68 \mathrm{GeV} / \mathrm{c}$, i.e. the reconstructed momentum value seems a little underestimated. The difference can be attributed to non-homogeneities of BM@N magnetic field [5].

\section{Summary}

1. The complex method of particle hit and track reconstruction in the drift chambers is tested on BM@N deuteron beam data.

2. The $r-t$ calibration curve is estimated and applied to calculate DCA coordinates of the DCH hits.

3. DCH hits are estimated under the assumption of track linearity. Hits in both the DCHs are aligned and fitted to produce linear track candidates. 

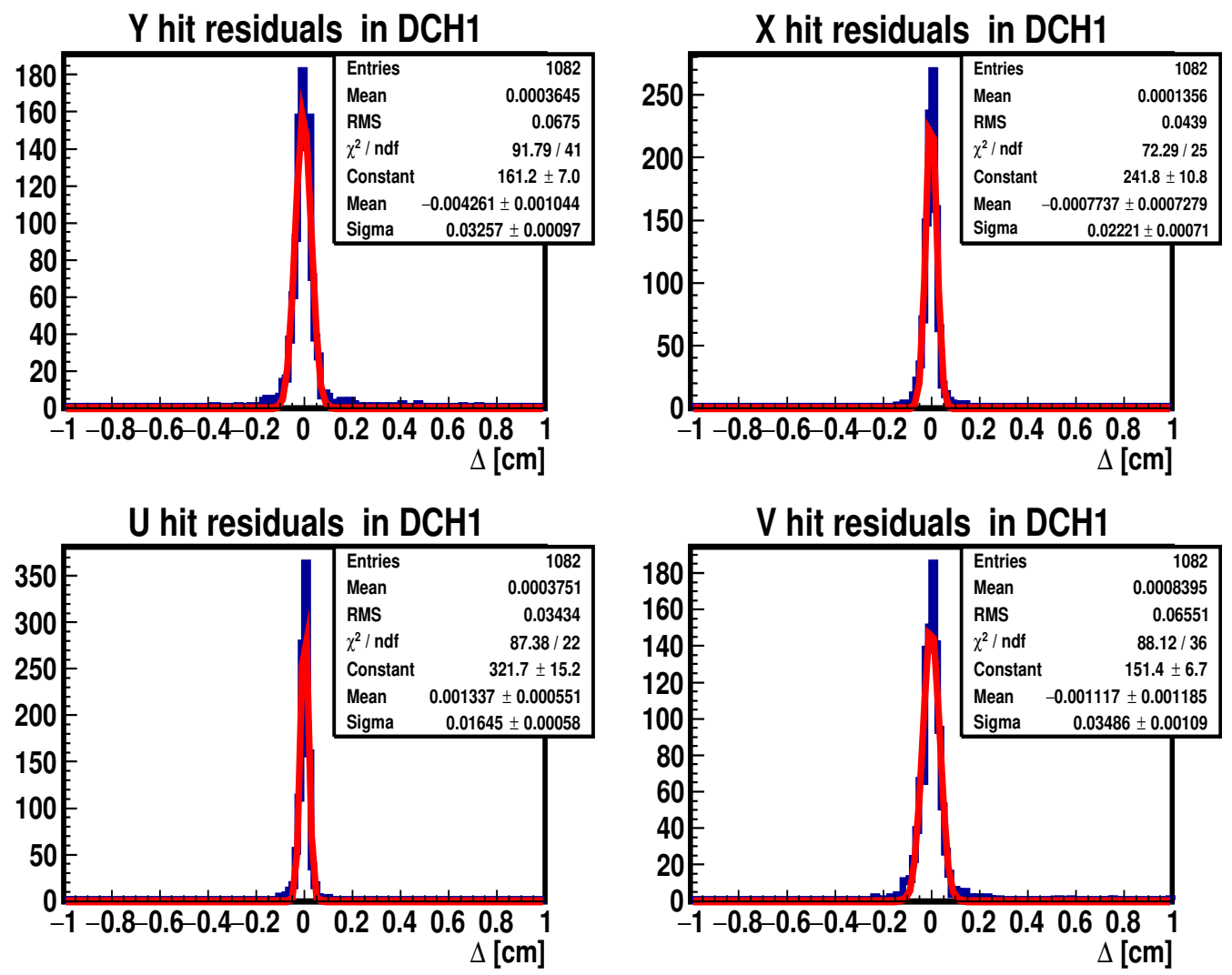

Figure 4. Hit residuals in all the DCH1 coordinate planes after the last iteration.
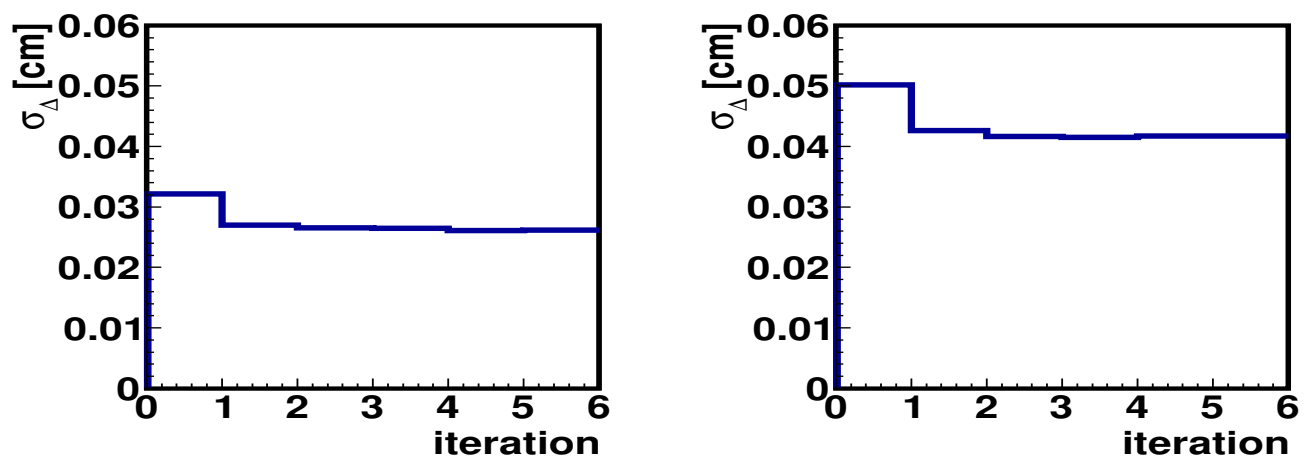

Figure 5. Gaussian $\sigma$ of residual spectra for DCH1 (left) and DCH2 (right).

4. The hit and track reconstruction is optimized by the autocalibration method. 

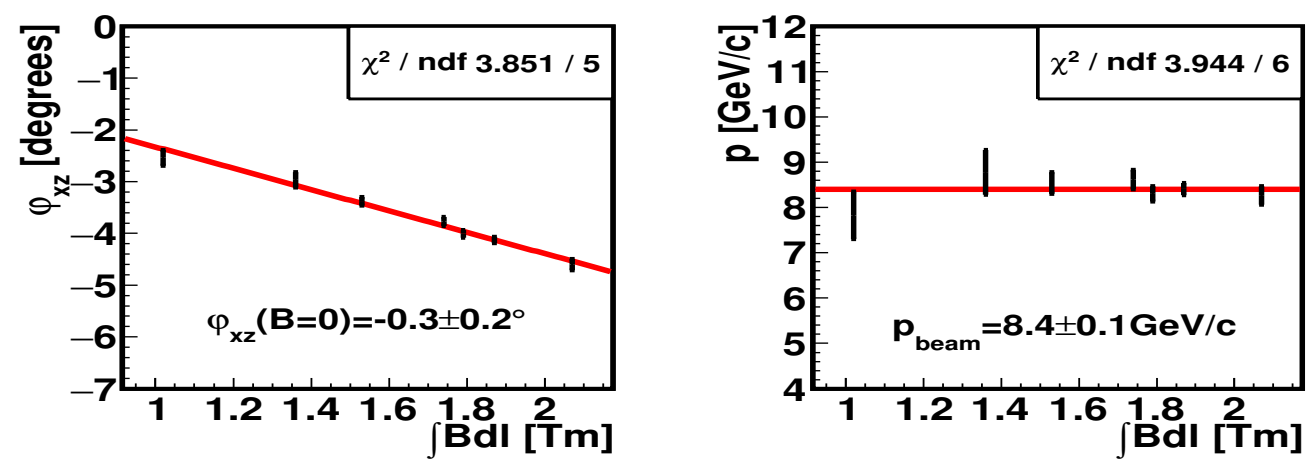

Figure 6. Deflection angle of deuteron beam in $x z$ plane (left) and deuteron momentum (right) as functions of magnetic field integral.

5. The DCH hit residual spectra fitted by Gaussian functions imply the coordinate resolutions ranging from $\sigma \approx 160 \mu \mathrm{m}$ up to $570 \mu \mathrm{m}$ for different DCH planes.

6. The verification test based on the beam momentum estimation proves a reliability of the proposed method, however some refinement is still desirable.

\section{References}

[1] T.O. Ablyazimov et al., BM@N Collaboration, BM@N-Baryonic Matter at Nuclotron (Conceptual Design Report, 2012). http://nica.jinr.ru/files/BM@N/BMN_CDR.pdf

[2] M. Kapishin, Eur. Phys. J. A 52, 213 (2016)

[3] J. Fedorišin, EPJ Web of Conferences 108, 02021 (2016)

[4] D. Béderéde et al., Nucl. Instrum. Meth. A 367, 88-91 (1995)

[5] P.G. Akishin et al., physics.ins-det arXiv:1407.7096v1 (2014)

[6] G. Avolio et al., Nucl. Instrum. Meth. A 523, 309-322 (2004)

[7] W. Erni et al., Eur. Phys. J. 49, 25 (2013)

[8] V. Glagolev et al., Instrum. and Exp. Tech. 56, 387-397 (2013)

[9] Tables of Physical $\mathcal{F}$ Chemical Constants (16th edition 1995) (Kaye \& Laby Online, version 1.0, 2005). http://www.kayelaby.npl.co.uk 